\title{
Crotalaria lanceolata (Leguminosae - Faboideae): A new record for India
}

\author{
K. Ravikumar ${ }^{1 *}$, N.M. Ganesh Babu ${ }^{1}$ and A.C. Tangavelou ${ }^{2}$ \\ ${ }^{1}$ Centre for Conservation of Medicinal Resources, The University of Trans-Disciplinary Health Sciences and \\ Technology, 74/2, Jarakabande Kaval, Attur P.O., Yelahanka via, Bengaluru, Karnataka - 560 064, India. \\ ${ }^{2}$ Department of Plant Biology and Plant Biotechnology, Guru Nanak College (Autonomous), Guru Nanak \\ Salai, Velachery, Chennai, Tamil Nadu - 600 042, India. \\ *Email: ravikumarfrlht@gmail.com
}

\begin{abstract}
Crotalaria lanceolata E. Mey., a pantropical species collected from Mandya district of Karnataka is reported for the first time from India. Detailed description, notes on distribution and photographs are provided for easy identification.
\end{abstract}

Keywords: Crotalaria lanceolata, India, Karnataka, new record.

\section{Introduction}

Crotalaria L., one of the largest genera of Leguminosae comprises c. 700 species, is chiefly distributed in tropical and subtropical regions of the world (Polhill, 1982; Mabberley, 2017). Ansari (2008) recorded 93 species, 1 subspecies, 17 varieties and 2 formae in India. In southern India, it is represented by 67 species and 8 infraspecific taxa (Sibichen \& Nampy, 2007; Jabbar et al., 2010; Krishnaraj et al., 2011; Krishnaraj \& Mohanan, 2012; Binu et al., 2012). During a survey of medicinal plants in Mandya district of Karnataka state, the second author collected an interesting specimen of Crotalaria (only two individuals were found) with trifoliate, linear-lanceolate leaflets. Careful examination with the relevant literature (Baker, 1871; Drummond, 1972; Pope et al., 2003; Ansari, 2008; Li et al., 2010; Leverett \& Woods, 2012; Garcia et al., 2013; Tiwari \& Ansari, 2014; Flores \& Tozzi, 2018) and comparison with type specimens, it was identified as Crotalaria lanceolata E. Mey. and is reported here as a new record to India.

Crotalaria lanceolata E. Mey., Comm. Pl. AfricaAustralia 1: 24. 1836; Baker in Oliver et al., Fl. Trop. Africa 2: 36. 1871; R.B. Drumm., Kirkia 8(2): 218. 1972; Pope et al., Fl. Zambesiaca 3(7): 140. 2003; J.Q. Li et al. in Z.Y. Wu et al., Fl. China 10: 106. 2010; Leverett \& Woods, Castanea 77(4): 371. 2012; Garcia et al., R. Bras. Bioci., Porto Alegre 11(2): 214. 2013; A.S. Flores \& Tozzi, Phytotaxa 346(1): 42.
2018. C. mossambicensis Klotzsch, Naturw. Reise Mossambique 6: 60, t.10. 1861.

Fig. 1.

Annual herbs up to $1.25 \mathrm{~m}$ tall. Stems erect, branched, terete, ribbed, pilose when young, glabrescent when mature. Leaves 3-foliolate; petioles 3-5 cm long; petiolules $1-2 \mathrm{~mm}$ long; leaflets linear to linear-lanceolate, $4-14 \times 0.5-1.5$ $\mathrm{cm}$, sparsely pilose or glabrous, cuneate at base, acuminate or mucronate at apex, entire along margins. Stipules absent. Inflorescence a terminal raceme, upto $20 \mathrm{~cm}$ long, 10-40-flowered. Pedicels 3-5 mm long. Bracts subulate to filiform, 0.5-3 $\mathrm{mm}$ long, expanded at base. Bracteoles subulate, narrow, $0.3-1 \mathrm{~mm}$ long inconspicuous, persistent, normally inserted at base of calyx or rarely on pedicels. Calyx sub-campanulate, c. $3 \mathrm{~mm}$ across, 5-lobed, pubescent; lobes triangular, c. $1 \mathrm{~mm}$ long, pubescent, shorter than tube. Petals 5, yellow with reddish-purple veining outside; standard petal broadly elliptic to orbicular, c. $1 \mathrm{~cm}$ long, with 2 appendages below; wing petals oblong, $c .1 \mathrm{~cm}$ long, marginally pilose at base; keel petals $c .1 \mathrm{~cm}$ long, rounded at apex, margins pubescent, beak long. Anthers dorsifixed, 1.3-1.6 mm long. Ovary 5-6 mm long, stipitate, glabrous; style curved. Pods oblong, narrowly cylindrical, curved at apex, $2-4 \times 0.5-1.5 \mathrm{~cm}$, glabrous, brown or black when mature; seeds c. 30, obliquely cordate, c. $2 \times 2 \mathrm{~mm}$; seed coat orange or red.

Flowering \& fruiting: June-September. 

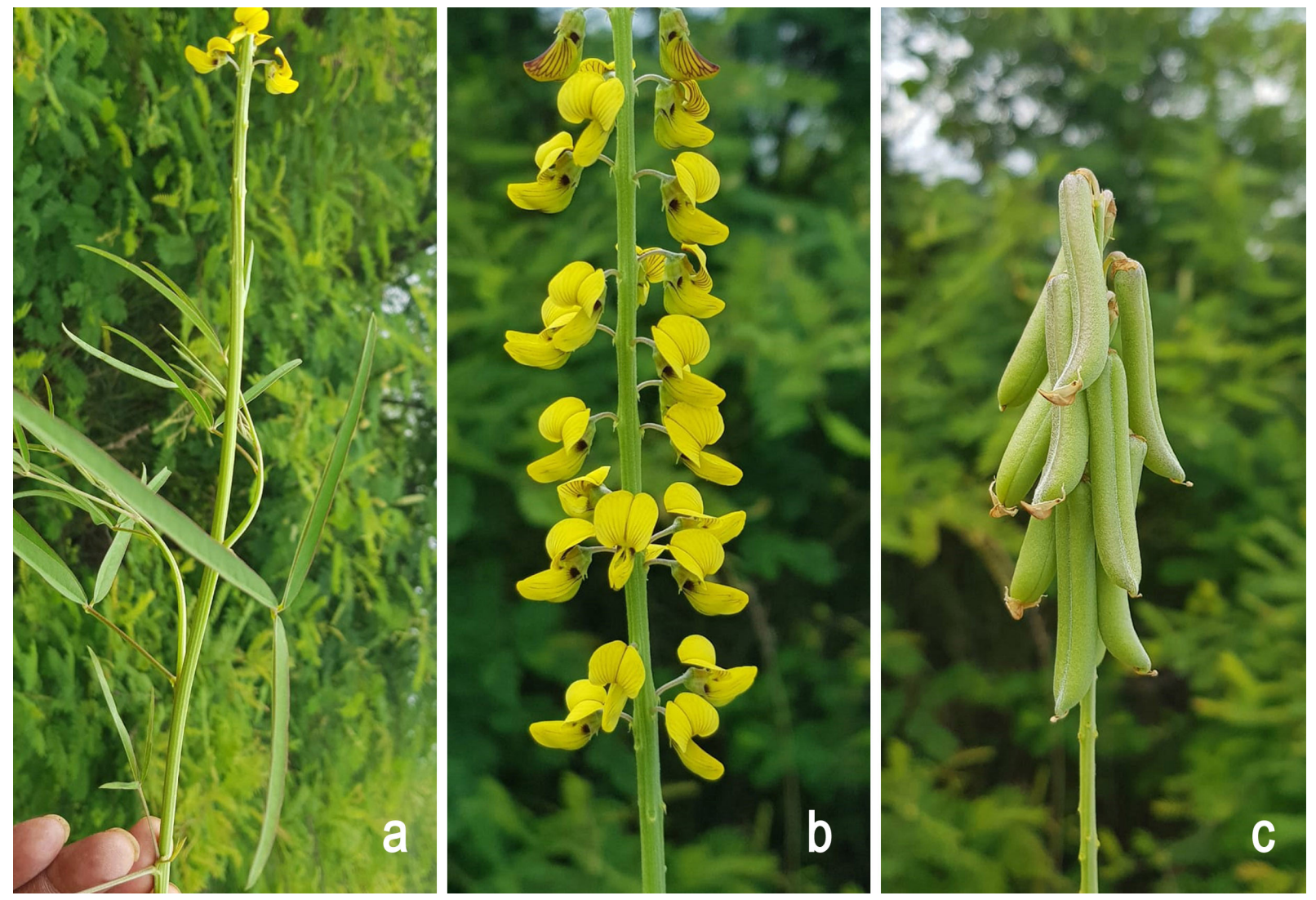

Fig.1. Crotalaria lanceolata E. Mey. a. A flowering branch; b. Closer view of Raceme; c. Closer view of Pods

Habitat: Growing in cultivated land at an altitude of $745 \mathrm{~m}$ along with Balanites aegyptiaca (L.) Delile, Crotalaria pallida Aiton, Endostemon viscosus (Roth) M.R. Ashby and Solanum surattense Burm.f.

Distribution: Native to Tanzania to South Africa, Madagascar. Introduced into Alabama, Bolivia, Brazil, China South-Central, China Southeast, Colombia, Florida, Honduras, Leeward Is., New South Wales, Paraguay, Queensland, South Carolina and Taiwan. (http://www.plantsoftheworldonline.org)

Notes: Authors could not locate any additional populations even after a careful search for this species in the vicinity, where the whole area is covered by a mono-dominant population of Crotalaria pallida Aiton. Most probably, this species might have entered the locality through horticultural trade, especially through the import of Water Melon [Citrullus lanatus (Thunb.) Matsum. \& Nakai.] seeds from Sudan for cultivation in Mandya district of Karnataka.

Specimens examined: INDIA, Karnataka, Mandya district, Mallasandra, $12^{\circ} 54^{\prime} 56.62^{\prime \prime} \mathrm{N} ; 78^{\circ}$ 50'25.51" E., 745 m, 22.06.2018, N.M. Ganesh Babu, S.K. Manjunatha, V.J. Umesh \& Hanumantharayappa 121203 (FRLH).

\section{Acknowledgements}

The authors are grateful to Shri Darshan Shankar, Vice Chancellor, TDU, Bangalore for providing facilities and support; Dr. A.A. Ansari (Botanical Survey of India) for confirming the identity and Prof. D. Narasimhan, Madras Christian College for critically going through the manuscript.

\section{Literature Cited}

Ansari, A.A. 2008. Crotalaria L. in India. Bishen Singh Mahendra Pal Singh, Dehra Dun.

Baker, J.G. 1871. Papilionaceae. In: Oliver et al. (eds.), Flora of Tropical Africa, Vol. 2. L. Reeve \& Co., London. pp.1-258.

Binu, T., Prabhu Kumar, K.M., Rajendran, A., George, S. \& I. Balachandran 2012. A new variety of Crotalaria ramosissima (Fabaceae) from Tamil Nadu, India. Asian Pacific J. Trop. Biomed. 2(3): S1412-S1414.

Drummond, R.B. 1972. A list of Rhodesian Legumes. Kirkia 8(2): 218.

Flores, A.S. \& A.M.G. Tozzi 2018. A synopsis of the genus Crotalaria (Leguminosae) in Brazil. Phytotaxa 346(1): 31-58. 
Garcia, J.M., Kawakita, K., Miotto, S.T.S. \& M.C. Souza 2013. O gênero Crotalaria L. (Leguminosae, Faboideae, Crotalarieae) na Planície de Inundação do Alto Rio Paraná, Brasil. R. Bras. Bioci., Porto Alegre 11(2): 209-226.

Jabbar, M.A., Krishnaraj, M.V. \& N. Mohanan 2010. Crotalaria incana subsp. purpurascens (Fabaceae), a new record for India. Rheedea 20: 131-132.

Krishnaraj, M.V. \& N. Mohanan 2012. A new combination in Crotalaria multiflora (Am.) Benth. (Fabaceae). Phytotaxa 44: 58-62.

Krishnaraj, M.V., Mohanan, N. \& V.T. Antony 2011. A new variety of Crotalaria assamica (Fabaceae: Papilionoideae) from the Western Ghats, India. Rheedea 21: 153-156.

Leverett, L.D. \& M. Woods 2012. The Genus Crotalaria (Fabaceae) in Alabama. Castanea 77(4): 371.

Li, J.Q., Sun, H. Polhill, R.M. \& M.G. Gilbert 2010. Crotalaria In: Wu, Z.Y., P.H.Raven \& D.Y.Hong (eds.), Flora of China, Vol. 10 (Fabaceae). Science Press, Beijing \& Missouri Botanical Garden Press, St. Louis. pp. 105-107.
Mabberley, D.J. 2017. Mabberley's Plant-Book: A portable dictionary of plants, their classification and uses, Fourth Edition. Cambridge University Press, Cambridge.

Polhill, R.M. 1982. Crotalaria in Africa and Madagascar. Royal Botanic Garden, Kew.

Pope, G.V., Polhill, R.M. \& E.S. Martins 2003. Flora of Zambesiaca, Vol. 3(7) (Papilionoideae: Loteae, Galegeae, Viceae, Cicereae, Trifolieae, Podalyrieae, Crotalarieae \& Genisteae). Royal Botanic Gardens, Kew, pp. 274.

Sibichen, M.T. \& S. Nampy 2007. Crotalaria kurisumalayana Sibichen \& Nampy (Fabaceae), a new species from India. Candollea 62(1): 105-108.

Tiwari, A.P. \& A.A. Ansari 2014. Crotalaria shuklae A.P. Tiwari \& A.A. Ansari (Fabaceae) - a new species from India. Taiwania 59(1): 54-58.

Received: 26.09.2018

Revised and Accepted: 24.12.2018 